Document downloaded from:

http://hdl.handle.net/10251/63799

This paper must be cited as:

González Parra, GC.; Villanueva Micó, RJ.; Ruiz Baragaño, J.; Moraño Fernández, JA. (2015). Modelling influenza A(H1N1) 2009 epidemics using a randomnetwork in a distributed computing environment. Acta Tropica. 143:29-35. doi:10.1016/j.actatropica.2014.12.008.

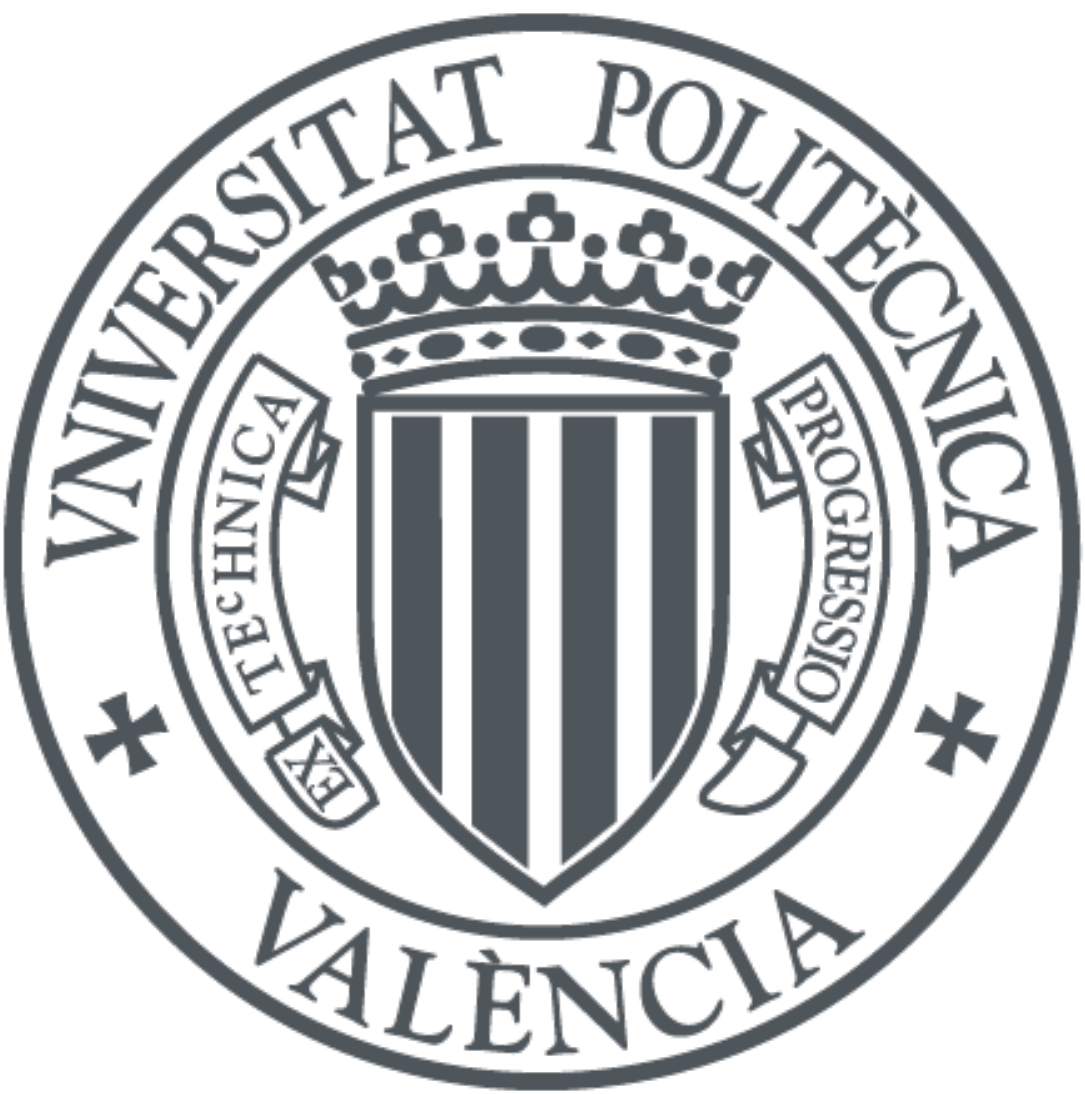

The final publication is available at

http://dx.doi.org/10.1016/j.actatropica.2014.12.008

Copyright Elsevier

Additional Information 


\title{
Modelling influenza A(H1N1) 2009 epidemics using a random network in a distributed computing environment
}

\author{
Gilberto González-Parra ${ }^{\mathrm{a}, \mathrm{b}, *}$ Rafael-J. Villanueva ${ }^{\mathrm{c}}$ \\ Javier Ruiz-Baragaño ${ }^{c}$ Jose-A.Moraño $^{c}$

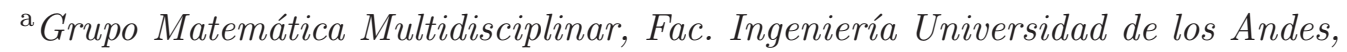 \\ Venezuela. \\ ${ }^{\mathrm{b}}$ Centro de Investigaciones en Matemática Aplicada (CIMA), Universidad de los \\ Andes, Venezuela. \\ ${ }^{\mathrm{c}}$ Instituto de Matemática Multidisciplinar, Universitat Politécnica de Valéncia \\ Edificio 8G, 2०, 46022 Valencia, Spain
}

\begin{abstract}
In this paper we propose the use of a random network model for simulating and understanding the epidemics of influenza $\mathrm{A}(\mathrm{H} 1 \mathrm{~N} 1)$. The proposed model is used to simulate the transmission process of influenza $\mathrm{A}(\mathrm{H} 1 \mathrm{~N} 1)$ in a community region of Venezuela using distributed computing in order to accomplish many realizations of the underlying random process. These large scale epidemic simulations have recently become an important application of high-performance computing. The network model proposed performs better than the traditional epidemic model based on ordinary differential equations since it adjusts better to the irregularity of the real world data. In addition, the network model allows the consideration of many possibilities regarding the spread of influenza at the population level. The results presented here show how well the SEIR model fits the data for the AH1N1 time series despite the irregularity of the data and returns parameter values that are in good agreement with the medical data regarding AH1N1 influenza virus. This versatile network model approach may be applied to the simulation of the transmission dynamics of several epidemics in human networks. In addition, the simulation can provide useful information for the understanding, prediction and control of the transmission of influenza $\mathrm{A}(\mathrm{H} 1 \mathrm{~N} 1)$ epidemics.
\end{abstract}

Key words: AH1N1/09 influenza epidemic, Random network model, Mathematical model, Distributed computing environment. 


\section{Introduction}

The pandemic virus AH1N1/09 is a flu virus of swine origin that was first identified in humans in April 2009 in Mexico and the United States [1]. The virus soon spread to the rest of the world and on June 11th, 2009, the WHO declared novel influenza $\mathrm{A}(\mathrm{H} 1 \mathrm{~N} 1)$ a pandemic [1]. The transmission of the virus AH1N1 occurs through effective contacts with an infectious individual.

Typical interventions include quarantine, isolation, travel restrictions, closing of public places, fear-based self quarantine and cancellation of events [1]. These interventions have economic costs to individuals and society related to lost work, increased school absenteeism and decreased business revenues $[1,2,3,4,5]$. It is important to mention that the entire vaccine production process takes at least six months to complete. However a pandemic influenza $\mathrm{A}(\mathrm{H} 1 \mathrm{~N} 1)$ vaccine became available in the U.S. in October 2009 [2].

Since there are more than 14,000 confirmed deaths worldwide caused by the AH1N1/09 virus, it is important to understand the dynamics of the AH1N1/09 virus. Every year approximately 36,000 people die from seasonal influenza or flu-related causes only in the U.S. [2]. Some models including the SEIR (Susceptible-Exposed-Infected-Recovered) model have been presented to study the dynamics of H1N1 influenza virus spread in different regions around the world $[6,7,3,8,9]$. The influenza virus has a latency (eclipse) stage and that is the main reason to choose the SEIR model over the SIR one $[10,11]$. It is important to remark that while some parameters of the SEIR model can be determined based on previous knowledge, other parameters must be estimated by fitting the model to the available data. Thus, fitting epidemiological mod-

els to real data becomes a central problem for the field of infectious disease epidemiology [12].

The study of contact networks, and more generally of social networks, is of growing importance in a diverse group of disciplines [13]. Simulating networks has the disadvantage of being computationally intensive: an epidemic in a population of just a few hundred thousand nodes can take hours of computation to obtain average results over a range of model parameters. However, that is a once-off cost of doing away with the assumption of a fully-mixed population, and the range of possibilities it provide makes it a necessary trade-off [14].

Usually, in the ODE models, all the factors involved in the transmission are embedded into the non linear model parameters. In the network models a disease spread structure is postulated and the transmission parameters can be interpreted as the probability of a successful contact among all the possible

\footnotetext{
* Author for correspondence

Email address: gcarlos@ula.ve (Gilberto González-Parra).
} 
contacts. In the random networks, in each step each individual (node) is studied and may be monitored over time in order to know its particular evolution. Additionally, in ODEs, seasonality is usually forced introducing a term involving a cosine, whereas, as has been shown in [15], constant parameters located in a certain range simulate naturally the seasonal disease behavior.

In general stochastic epidemic models are to be preferred over deterministic when their analysis is possible. In first place, a natural way to describe the spread of a disease is stochastic; one defines the probability of disease transmission between two individuals rather than rather than stating certainly whether or not the transmission will occur. On the other hand, deterministic epidemic models rely on the law of large numbers. Moreover, when considering extinction this can only be analysed with stochastic models [16].

To the best of our knowledge very few antecedents of mathematical models based on networks have been used to study the dynamics of some real world epidemics with infected populations. Some interesting previous work is: a hierarchical network model for the epidemic of $\mathrm{A} / \mathrm{H} 1 \mathrm{~N} 1$ virus spreading in Romania [17], a network model to study the RSV spread in the population of the Spanish region of Valencia [15,18], a scale-free network modeling dengue in Singapore [19], a complete graph network modeling the social obesity epidemic [20], a social network to investigate the 2007 outbreak of equine influenza in Australia [21], a network study of a measles outbreak in Hagelloch, Germany, in 1861 consisting of 188 affected individuals [22] and a network model that describes the empirical data of the 2000/2001 cholera epidemic which took place in the Kwa Zulu-Natal Province, South Africa [23]. In [24] authors developed an interesting agent-based model, in which each individual is explicitly represented and vector populations are linked to precipitation estimates. They implemented the model on both scale-free and regular networks.

It is important to remark that the classical SEIR epidemic model does not reflect the irregularity (random fluctuations) of the AH1N1 real data regarding infected population. In addition, the deterministic model describes the epidemic using mean values without considering the population variance or that the population has discrete values $[25,26,24]$. However, the SEIR model can also be used with contact networks and the data simulation results are non-smooth due the underlying discrete structure of the network model. The simulation results using network models may be very different than the counterpart deterministic model $[15,25,26]$.

Social networks have been constructed using real data and used to study some health issues [20,27]. For instance, the monitoring of individual activities and construction of the network has been attempted for the city of Portland [28]. The spread of several infectious diseases is determined by random encounters among people who live in the same geographical area: meeting at the bus 
stops, crossing the streets, gathering at shopping centers, etc. The transmission of the AH1N1 virus is possible through effective contacts with an infectious individual. In this research we consider that the random network model is the most appropriate to the modeling of the transmission of the AH1N1 virus.

In order to obtain the simulation results for the network SEIR model in the shortest time a client-server application was developed to coordinate many computers in the calculation of the epidemic propagation. Results were stored in the server for later processing. We have obtained more than one million tests for different combinations of the average number of contacts, $k$, and the transmission rate $\beta$. It is important to remark that $k$ and $\beta$ are constants to be determined by fitting the model to the real data of AH1N1 from the Venezuelan Nueva Esparta state during the year 2009.

In this work, we develop a network model to reproduce the dynamics of AH1N1 virus in the Venezuelan state of Nueva Esparta (mainly one island). To do that, we consider a SEIR model using a network framework to explain the pandemic dynamics. It should be mentioned that Nueva Esparta is a relatively large state with a low population density where continuous SEIR models did not succeed in explaining some AH1N1 real data dynamics [29,30]. Once the model is formulated, we use the Particle Swarm Optimization (PSO) algorithm in a distributed computing environment to determine the unknown model parameters that best fit to the reported data of Nueva Esparta with regard to infected individuals by AH1N1. The PSO algorithm was inspired by the social behavior of biological organisms, specifically the ability of groups of some species of animals to work as a whole in locating desirable positions in a given area, e.g. birds flocking to a food source $[31,32]$. Generally in the PSO method, particles move through the search space using a combination of an attraction to the best solution that they individually have found, and an attraction to the best solution that any particle in their neighborhood has found [31,32]. Thus, the PSO allows to search the optimum of a nonlinear problem. In this work the PSO is used in the fitting process in order to find the optimum values of the parameters $\beta$ and the scale factor $s$ that minimize the mean square error (MSE) between the real reported infected cases and the predicted infected cases of the network model. We select PSO as the optimization method since we consider that is suitable when the results that must be optimized depends on several parameters and depends on random processes. Nevertheless, several other optimization methods can be used but this topic is out of the scope of this study.

Similar works has been developed previously. For instance, in [33] authors study the pandemic (H1N1) 2009 influenza in two states of Australia using as a tool a SEIR stochastic model in order to reflect the variability of the outbreak. Additionally, in [34] authors estimated reproduction numbers using a stochastic approach and computer-generated data. 


\section{Methods}

The reported cases of pandemic AH1N1 influenza represent those who tested positive to influenza AH1N1 virus using laboratory testing and the data is reported by weeks. The data was originally collected from state health institutions where individuals presenting acute symptoms were assisted. The reported dates correspond to when the samples were taken. The influenza AH1N1 tests were performed using specimens from nasal swabs or aspirates and real-time reverse transcription polymerase chain reaction (RT-PCR) or viral culture. The reported positive cases of pandemic AH1N1 influenza in Venezuelan Nueva Esparta state are depicted graphically in Figure 1. The highest number of positive reported cases was in the 34th week of 2009. The data was provided by the National Health Department of Venezuela. First row corresponds to week number of year 2009. It is important to remark that the number of infectious by AH1N1/09 detected in each week is because these people went to see a doctor and were reported. Data about deaths are not available.

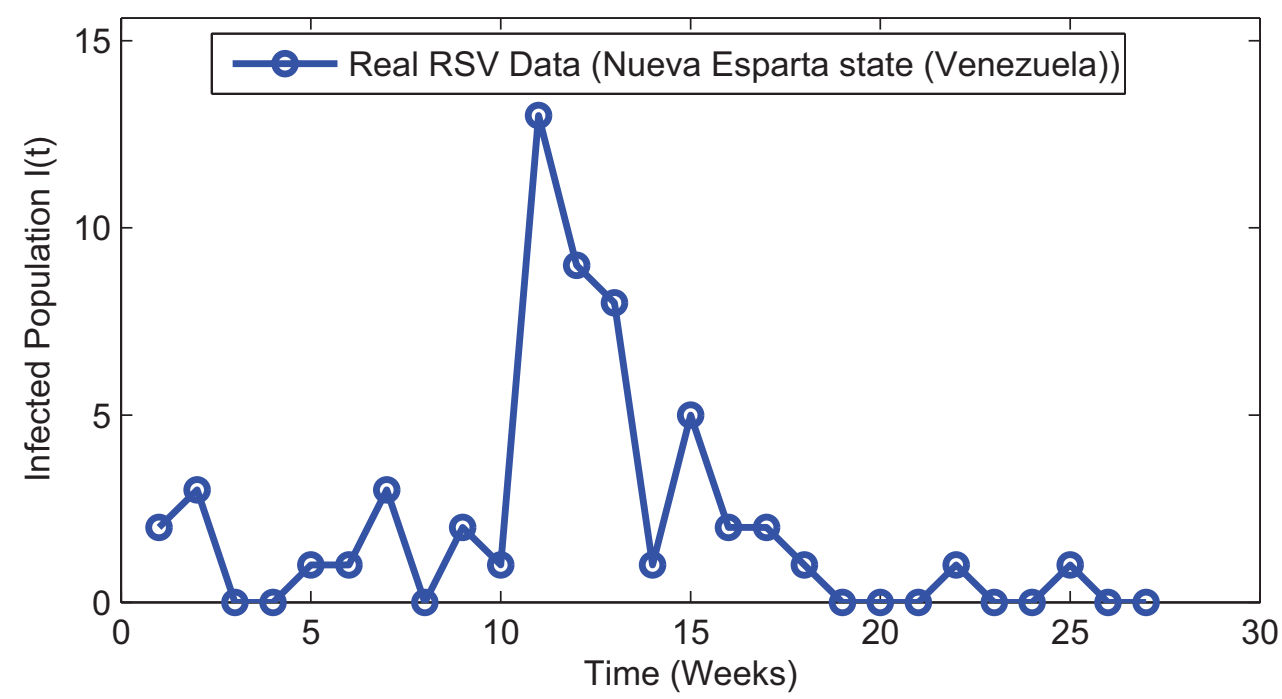

Figure 1. Graph of the data of reported positive cases of pandemic AH1N1 influenza in Venezuelan Nueva Esparta state.

Here we introduce the proposed SEIR network model considering births and deaths. The SEIR epidemiological model considers that the whole population $N(t)$, is divided into four subpopulations: $S(t)$ susceptible, $E(t)$ exposed people incubating the virus, infectious $I(t)$ and recovered subpopulation $R(t)$. In addition, we consider that newborn children become susceptible at rate $\mu$ (birth rate) and individuals leave the system at rate $d$ (death rate). Diseaserelated deaths are disregarded since they are much smaller than natural deaths and are not a main driving factor on the dynamics. In particular, a US study estimated a death rate of 4 deaths per 100000 population during April 2009- 
April 2010 [35]. An individual in $S(t)$ flows to $E(t)$ because people in $I(t)$ transmit AH1N1 by effective contacts at rate $\beta$. Finally we consider that once an individual is recovered, he acquires permanent immunity [2]. In this model the infected individuals recover at rate $\rho$, where $1 / \rho$ is the average time to recover from AH1N1 influenza. In addition, exposed individuals become infectious at rate $\omega$, where $1 / \omega$ is the average time to become infectious. The model diagram is depicted in Figure 2.

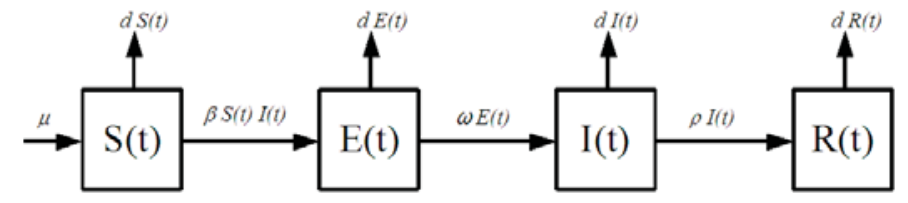

Figure 2. Flow diagram of the SEIR mathematical model for AH1N1 transmission dynamics.

Using the above description, we build a random network model to describe the evolution dynamics of the spread of AH1N1.

First, we build the network. Let us take $N=450138$ the number of network nodes as the population of Nueva Esparta. Let $k$ the average number of links of a node, also called degree of a node. $k$ is unknown and not necessarily an integer, but its value may determine the speed of the disease spread. Therefore, the network has $N \times k / 2$ links, ties or undirected edges to be assigned randomly as follows:

(1) For $i=1$ to $N \times k / 2$.

(a) Take randomly two different values between 1 and $N$, say $i_{1}$ and $i_{2}$.

(b) If the link $\left(i_{1}, i_{2}\right)$ exists, repeat the step (a). Else, include $\left(i_{1}, i_{2}\right)$ in the link list.

Using the above algorithm, as shown in [15], the node degrees follow a Poisson distribution. Also, note that different runs of the above algorithm produce different networks with average node degree $k$.

Now, we establish the initial condition of the network respect to the disease. The initial conditions regarding the topology of the network and the number of infected can affect the dynamics of the epidemic [36]. Since initial conditions are not known, we use different values for the initial infected and exposed individuals in order to generate with the network model a dynamics as close as possible to the real data. In this way, all the $N$ nodes will be labelled as susceptible except a random number between 0 and 99 labelled as exposed and a random number between 1 and 99 labeled as infectious. It is important to remark that in all the initial scenarios the total population $\mathrm{N}$ is the same. The initial time is taken as the Monday of the 24th week of 2009.

It is time to define the model evolution rules. The evolution will be carried 
out daily. Let $\mu$ and $d$ be the birth and death rates in Nueva Esparta. The unknown model parameters we are going to use as model input are:

- $k$, average nodes degree,

- $\beta$, disease transmission rate,

- $\omega$, inverse average time an exposed becomes infectious,

- $\rho$, inverse average time an infectious becomes recovered.

The incubation period for pandemic AH1N1 virus has been reported to be 2-10 days with a mean of 6 days [1]. Therefore, the mean time in the exposed class $E(t)$ has been assumed such that $\omega=\frac{1}{6}$ days $^{-1}$. The infectious period has been reported between four and seven days with $\rho=\frac{1}{7}$ days $^{-1}[37,38]$. However, in young children and in immunocompromised or severely ill patients, the infectious period might be longer [39].

In the following, rnd() denotes a function that returns a random number between 0 and 1 .

Thus, the network evolves as follows (take into account that $N$ changes over time):

- For every day from the Monday of the 24th week of 2009 until the Sunday of the 50th week, with daily jumps:

- If there are no exposed or infectious, the simulation finishes, because nobody can be infected.

- New births. $N \mu / 365$ is the average daily births. For each node and each day, calculate the first value of $h$ such that

$$
\operatorname{rnd}()<\sum_{i=0}^{h} e^{-k} k^{i} / i !
$$

(follows a Poisson distribution), where $h$ is the number of links of the newborn. Then, take randomly $h$ different values between 1 and $N$ and build the new links.

- Deaths. $N d / 365$ is the average daily deaths. For each node and each day, calculate the first value of $h$ such that

$$
\operatorname{rnd}()<\sum_{i=0}^{h} e^{-k} k^{i} / i !
$$

(follows a Poisson distribution), being $h$ is the number of links of the node. Then, take randomly $h$ different values between 1 and $N$ and remove the nodes and their links.

- For every node 
If the node is infectious and $h$ is the number of days it is infected, if

$$
\operatorname{rnd}()<\sum_{i=0}^{h+1} e^{-\rho} \rho^{i} / i !
$$

(follows a Poisson distribution), the node becomes recovered and it and its links are removed from the system.

If the node is exposed and $h$ is the number of days it is exposed, if

$$
\operatorname{rnd}()<\sum_{i=0}^{h+1} e^{-\omega} \omega^{i} / i !
$$

(follows a Poisson distribution), the node becomes infectious.

If the node is susceptible, for every infectious node linked with it, if $\operatorname{rnd}()<\beta$, the node becomes exposed.

\section{Results}

This section is devoted to the presentation of the simulations and fitting results of the SEIR random network model in order to explain in a more suitable way the irregularity of the time series real data regarding the AH1N1 virus. In addition, results for the classical SEIR model are presented in order to compare with the SEIR random network model.

\subsection{Random network model fitting}

Here, it is presented the simulation and fitting results of the SEIR random network model in order to explain in a more suitable way the irregularity of the time series real data regarding the AH1N1 virus. Once the procedure to simulate the transmission dynamics of AH1N1 in Nueva Esparta has been implemented, we are able to find out the model parameters in order to fit the model with the reported data of infected cases depicted in Figure 1. Simulations are performed using the Monte Carlo method, assuming constant population and connections.

It is important to remark that the differences between time points regarding real data of AH1N1 infected population in Nueva Esparta state is small and the classical SEIR epidemic model is not able to reproduce this dynamic. For instance in [15] the authors used a network model to simulate a epidemic of RSV with a larger infected population. In this way using a network model to reproduce this profile is a challenging problem and our results are scalable to larger populations. 
To do this, as a first approach, we carried out around 480.000 Monte Carlo simulations taking $\mu=d=17.16$ per thousand (we consider birth and death rates equal because the short time, 25 weeks, simulated), $0.001<\beta<1$, $1<k<50,4<\omega^{-1}<8,5<\rho^{-1}<9,1$ initial infectious and $0,1,2,3,4$ or 5 exposed. With this first approach we try to reduce the range of parameters to find the best fitting.

To carry out the 480.000 simulations in a reasonable time, we tackle the problem by using a computational system following the paradigm of distributed computing that allows us to estimate the parameters in the random network epidemic model. This paradigm consists of a client/server structure where the server delivers tasks to be carried out by client computers, and when the task is finished, the client sends the obtained results to the server to be stored until all tasks are finished and ready to be analyzed. In our case, every task consists of a network model simulation with a set of parameters and the results are persons in each disease state at any time. Among all the simulations carried out, all the estimated parameters that make the best fitting are,

- $k=13$,

- $\beta=0.014163$,

- $\omega=\frac{1}{5}$,

- $\rho=\frac{1}{7}$,

- 1 exposed,

- 1 infectious,

- scale, $s=1$,

with mean square error equal to 1.567 . It should be mentioned that the fitting process gives optimum results with connectivity degrees around $k=13$. This fitting, not being poor, nevertheless has some drawbacks, such as that the highest peak is not reached and that there is a second lower peak not represented in the numerical simulation of the network model. Thus, a better fitting is needed. It is important to remark, that in this case the network model was not able to reproduced the second peak since we restricted the mean exposed and recovery days just to integer values. However, in order to improve the network fit, we realized that exposed and recovery days may be real numbers since from a clinical point of view is correct to state that for instance exposed days are 3.5 (84 hours). In fact this assumption is assumed implicitly in the classical SEIR model since the transition between exposed and infected classes are assumed to follow an exponential continuous probability distribution.

It is important to remark, that in the network model the number of exposed and infected people, are represented by relatively small integer values, which makes the model sensitive to the initial conditions due to the disease extinction outcome. However, this is a tradeoff that needs to be paid in order to have a more realistic stochastic model [16]. 
In order to improve the fit and reproduce the second peak, we use the Particle Swarm Optimization algorithm, but in a more restricted parameter space based on the previous fitting process [31]. In addition, as has been mentioned above we include now noninteger parameter values for $k, \rho$ and $\omega$ and, we have opened the range for the initially infectious between 1 and 100 and for the initially exposed between 0 and 100 in order to be able to reach a better fitting. For this second approach, the best fitting, obtained using again the computing distributed system, is given by the estimated parameters

- $k=21.5$,

- $\beta=0.00849$,

- $\omega=\frac{1}{5.9}$,

- $\rho=\frac{1}{6.665}$,

- 3 exposed,

- 1 infectious,

- the scale $s=0.965$,

with mean square error equal to 1.446 . Notice that the fitting has been improved and include now noninteger parameter values for the optimum solution. In Figure 3 is presented graphically one fitting of the random network model to the time-series data of confirmed cases of pandemic AH1N1/09 influenza from the Venezuelan Nueva Esparta state. The simulation results of the SEIR random network model generate irregular time series that approach the real data. It is important to notice how good the fitting process is despite the irregularity of the data and the stochastic process underlying the proposed SEIR network model. The best fitted random networks have values around $\omega=\frac{1}{5}$ and $\rho=\frac{1}{7}$, which are in good agreement with the medical data regarding AH1N1 influenza virus $[1,37,38]$.

It should be mentioned that the fitting process gives optimum results with connectivity degrees around $k=21.5$. This fact reflects that the connectivity of the network is important and should be included in a realistic epidemiological model. On the other hand, it should be mentioned that $\beta$ also plays an important role of the spread of the disease, but its variation is less than the connectivity since depends mainly on the virus infectivity and environments conditions. For instance, in high density cities or regions, the connectivity $k$ would be greater than in Nueva Esparta state, and disease spread would be faster $[28,20]$.

\subsection{Model fitting using the classical SEIR epidemiological model}

In this section we perform a data fitting using the classical SEIR epidemiological model based on differential equations in order to compare with the 


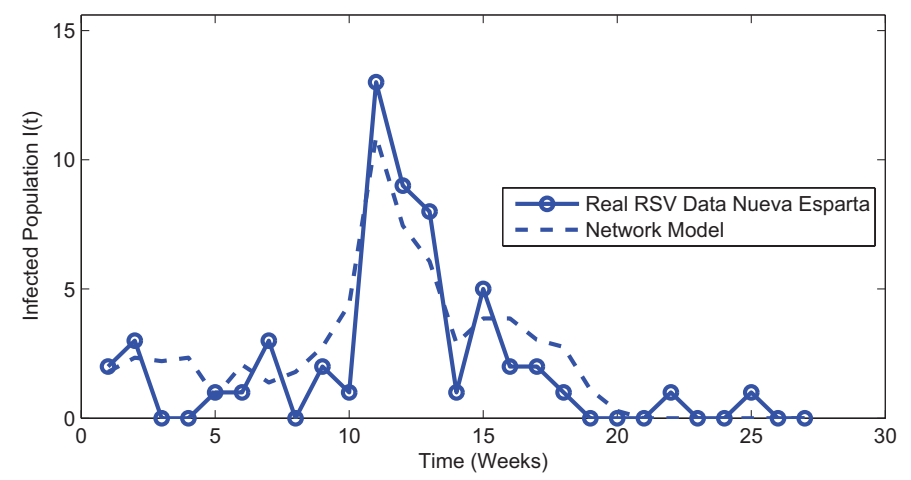

Figure 3. Model fitting for the Venezuelan Nueva Esparta state with the Particle Swarm Optimization algorithm of the SEIR random network model for the infected population. Real RSV data (line) and network SEIR model (dash line). The parameter values are $k=21.5, \beta=0.00849, \omega=\frac{1}{5.9}, \rho=\frac{1}{6.665}, 3$ exposed and 1 infectious as initial condition and a scale value of $s=0.965$.

network model fitting. The parameter values of the classical SEIR model based on differential equations need to be set. Since the epidemiology of pandemic AH1N1 virus is not accurately known or is not the same for each individual, the parameter values were chosen based on the best available data. It is important to remark that each one of the parameters $\omega$, and $\rho$, can be interpreted as the mean of the inverse length of the transit period between two sub-populations. Therefore the above numerical values computed for each parameter should be considered as average length of transition periods between two sub-populations and should not be regarded as a fixed time after which each individual crosses to a new sub-population. For instance with a fixed valued for the incubation period $\omega$, implicitly it is assumed different incubation periods varying between $[0, \infty]$. This is one of the main disadvantages of the classical continuous models. Moreover, in the real world the incubation period varies depending on several variables including the immune system of each individual. Thus, it has been argued that network models can be more realistic since includes these variations due to different factors. However, analytic analysis is more complex in comparison with the classical epidemiological models. Additionally, since we use the SEIR model for a relatively short time period we assume a constant population size. The population-scaled SEIR model (without loss of generality) is given by:

$$
\begin{aligned}
\dot{S}(t) & =\mu-d S(t)-\beta S(t) I(t), \\
\dot{E}(t) & =\beta S(t) I(t)-(d+\omega) E(t), \\
\dot{I}(t) & =\omega E(t)-(d+\rho) I(t), \\
\dot{R}(t) & =\rho I(t)-d R(t) .
\end{aligned}
$$


The time-series data of confirmed cases of pandemic AH1N1 influenza do not correspond exactly to subpopulation $I(t)$, since only a fraction $s$ (scale factor to be determined) of people who felt sick and decided to go to the doctor who reported the case. Additionally, some infected individuals do not show complete symptoms. In this way the parameters to be estimated by a fitting process to real data are the influenza AH1N1 transmissibility $\beta$ and the scale factor $s$.

The initial conditions in $t_{0}, S\left(t_{0}\right), E\left(t_{0}\right), I\left(t_{0}\right)$ and $R\left(t_{0}\right)$ are unknown. We assume that only a small fraction $(1 / 1000)$ of the population is infected in order to simulate the initial stage of the influenza epidemic and we set the initial conditions $S\left(t_{0}\right)=1-0.001, I\left(t_{0}\right)=0.001, E\left(t_{0}\right)=0$ and $R\left(t_{0}\right)=0$.

Thus, the fitting process is performed to estimate $\beta$ and the scale factor $s$ by least squares method and using Nelder-Mead algorithm. In order to compute the best fitting, we implemented the function

$$
\begin{aligned}
\mathbb{F}: & \mathbb{R}^{2} \longrightarrow \mathbb{R} \\
(\beta, s) & \longrightarrow \mathbb{F}(\beta, s)
\end{aligned}
$$

where $\beta$ and $s$ are variables such that:

(1) For a given $(\beta, s)$, solve numerically the system of differential equations (1) and obtain a solution $\hat{Y}_{i}(t)=\left(\hat{S}_{i}(t), \hat{E}_{i}(t), \hat{I}_{i}(t), \hat{R}_{i}(t)\right)$ which is an approximation of the real world solution $Y(t)$.

(2) Set $t_{0}=23$ (fitting process starts at week 23) and for $t=24,25, \ldots, 51$, corresponding to weeks where data is available, evaluate the computed numerical solution for subpopulation $I(t)$; i.e. $\hat{I}(24), \hat{I}(25), \hat{I}(26), \ldots$, $\hat{I}(51)$.

(3) Compute the root mean square of the difference between $s \hat{I}(24), s \hat{I}(25)$, $s \hat{I}(26), \ldots, s \hat{I}(51)$ and infectious data. This function $\mathbb{F}$ returns the root mean square value(RMS) which is given by:

$\mathrm{RMS}=\sqrt{(s \hat{I}(23)-I(23))^{2}+(s \hat{I}(24)-I(24))^{2}+\ldots+(s \hat{I}(51)-I(51))^{2}}$.

(4) Find a global minimum for the root mean square value(RMS)using NelderMead algorithm.

Function $\mathbb{F}$ takes values in $\mathbb{R}^{2}(\beta$ and $s$ ) and returns a positive real number, the root mean square that measures the closeness of the scaled infectious population, provided by the model, to time-series data. Hence, we can try to minimize this function using the Nelder-Mead algorithm [40,41], that does not involve the computation of any derivative or gradient, impossible to know for function $\mathbb{F}$. In order to find a global minimum, we take 500 initial different points for Nelder-Mead algorithm in the domain $[0,10] \times[0,10000] \subset \mathbb{R}^{2}$. We stored all the minima obtained and, among them, the values of $\beta$ and $s$ that minimize the function $\mathbb{F}$. In other words the Nelder-Mead algorithm return the 
best value of $\beta$ and $s$ such gives the minimum of the root mean square value (RMS). For similar details regarding the fitting procedure for the classical SEIR model see [42]. It is important to mention that the beta of the SEIR model is not the same as the $\beta$ of the network one. It is important to remark that there is correlation between SEIR's $\beta$ and $k[43,20]$.

In Figure 4 the fitting of the classical SEIR model, based on differential equations is presented. The data is the time-series data of confirmed cases of pandemic AH1N1/09 influenza from the Venezuelan Nueva Esparta state. The mean square error (mse) for this fitting is equal to 5.32476. In this particular case one gets a scale factor $s=0.000102$ using the total population scale $N$ instead of the normalized population represented in the model (1). Since $\mathrm{s}=0.96$ the network model does not need a scale factor to predict the infected cases. However, the classical SEIR model needs the scale factor to predict to real infected cases.

As it can be seen in Figure 4 the numerical simulation of the classical SEIR model generates a smoothness time series that differs from the real data. Additionally, the model does not allow the infected population to totally die off. In Figure 5 it can be observed the fitting of the network and SEIR classical models in order to make a graphic comparison among them. Notice that the network model has a second peak of infected cases, but with the SEIR model it is not possible to obtain that second peak.

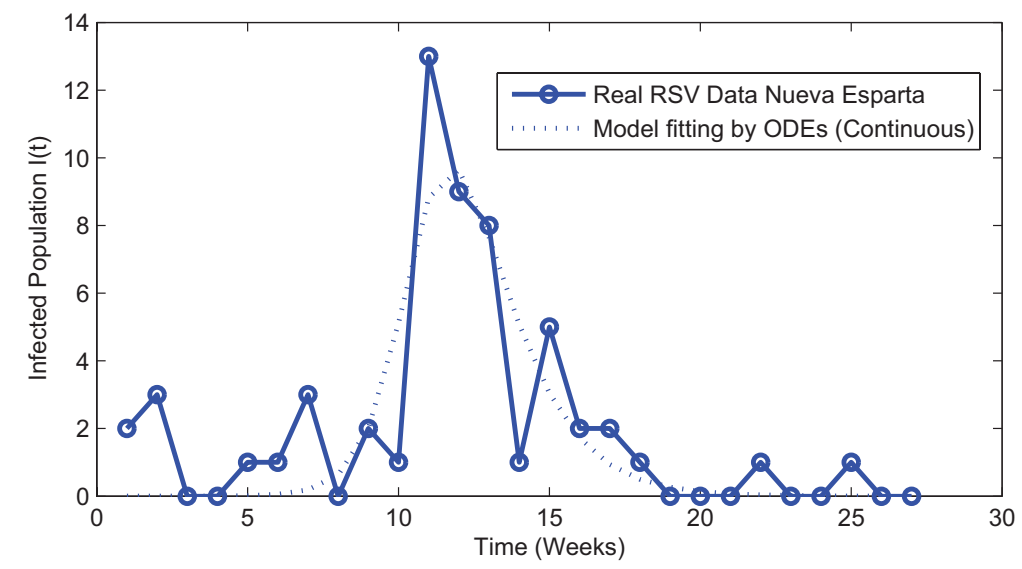

Figure 4. Model fitting for the Venezuelan Nueva Esparta state with the classical SEIR model. The influenza AH1N1 transmissibility $\beta=6.21$ and the scale factor $s=0.000102$.

\section{Discussion and conclusions}

In this paper it is proposed the use of a SEIR random network model approach for simulating and understanding some epidemics of influenza A(H1N1). The 


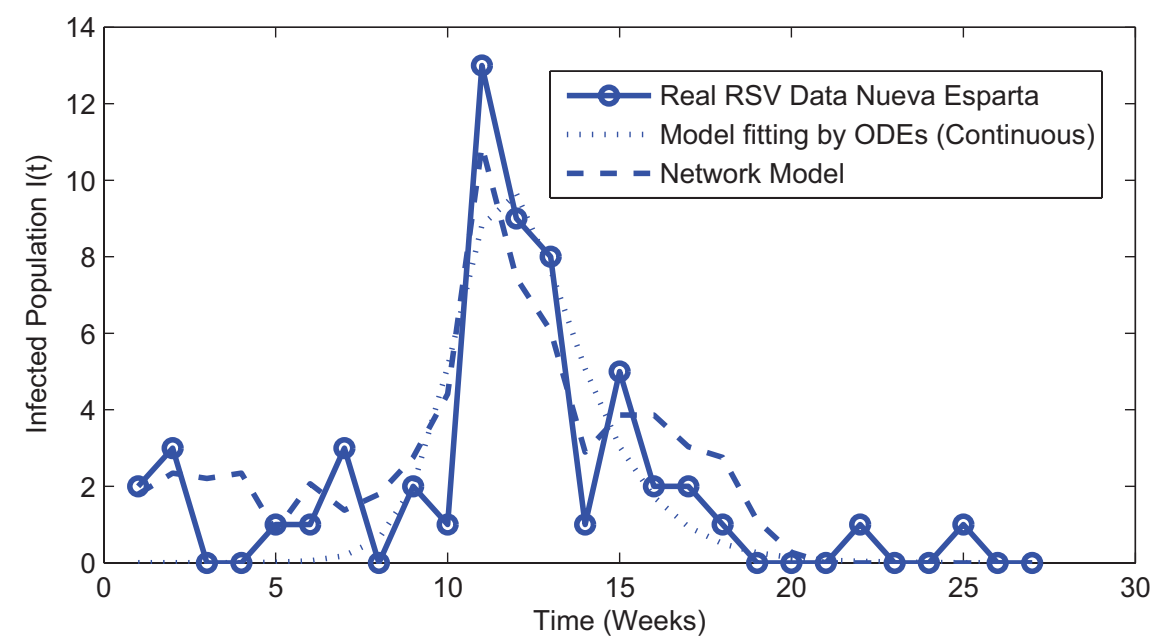

Figure 5. Model fitting for the Venezuelan Nueva Esparta state with the network and classical SEIR models. Real RSV data (line), network (dot-dashed) and classical SEIR model (points).

proposed model is used to simulate the transmission process of influenza $\mathrm{A}(\mathrm{H} 1 \mathrm{~N} 1)$ in a state of Venezuela using distributed computing in order to accomplish many realizations of the underlying random process.

The network model proposed here performs better than the traditional epidemic model based on ordinary differential equations since it reflects the irregularity of the real data and gives a better fit to the real data of reported infected cases. In addition, it is well known that more heterogeneity may be added to the network models in order to reproduce real world cases. The network model allows the consideration of many possibilities regarding the spread of the influenza at the population level. Furthermore, we show with these results the naturally observed strict extinction of the epidemic, which are not obtained with the SEIR classical differential equation model. In the network model more mean values for the transition periods were included in order to show more possible scenarios. On the other hand, using different mean values for the transition periods for the SEIR model the results were not significant different and we keep some standard values from the literature.

The fitting process of the SEIR network model to the AH1N1 infected population time series data provides parameter values that are in good agreement with the medical data regarding AH1N1 influenza virus. The fitting process returns a network connectivity degree around $k=21.5$ for Venezuelan Nueva Esparta region. However, in high density cities or regions, the connectivity $k$ would be greater than in Nueva Esparta state, and disease spread would be faster $[28,20]$. The algorithm used here for the network model can be modified in several ways and variations of our algorithm have been used in other work related to network models $[15,26,44]$. 
This versatile network model approach may be applied to the simulation of the transmission dynamics of several epidemics in human networks. In addition, the simulation can provide useful information for the understanding, prediction and control of the transmission of influenza A(H1N1) epidemics. For instance, if we would like to study the effect of a vaccination program it is possible to add to the network a new node class where the nodes can not transit to the infected class. Moreover, the network model can also be modified to include some health policies such as antiviral treatment which may be included by simply modifying the infectious period. In regard to future work and as has been suggested by other authors, models that include detailed information of the human contact network may be introduced in the model. This detailed information is not easy to collect but some interesting work has taken a first approach [21,28]. In addition, for influenza A(H1N1) infected population time series data from years 2010 it is necessary to include vaccinated population due to the fact that influenza $\mathrm{A}(\mathrm{H} 1 \mathrm{~N} 1)$ vaccine programs have been implemented in the studied regions. It can be mentioned that the models are a simplification of the complex real world where there are many variables that affect the dynamics of influenza, such as, school periods, weather or social events, but since real data regarding these factors are not available and in order to have a manageable model with reliable results it is necessary to disregard some of these factors.

The computational cost of network simulation is higher than the classical SEIR model. However, it is possible to obtain more reachable epidemic scenarios than with the classical SEIR epidemiological model. In this way, the network model could predict a higher number of infected cases and this may affect the plans and budget of health institutions. In some countries or regions this fact could be a very important health issue. Therefore, we think that network modeling decision would depend on the particular health authorities and how much risks they desire to take with regard to medicines, beds in hospitals, personal and all the variables involved in attending a epidemic.

We think that further research using complex networks is going to continue growing and more challenges are going to be faced in the future. However, an increasing amount of computing resources are needed in order to deal with this type of models. Several characteristics can be added to each node in order to represent the variability among individuals. Following these ideas more data need to be collected and also data mining techniques must be used for large data sets. This direction of research has been applied in several areas of bioscience, bioinformatics and bioengineering. Thus, we think that the proposed methodology or similar is going to be extended and be more useful in the near future.

\section{Acknowledgements}

First author has been supported by CDCHTA project I-1289-11-05-A. Third 
author acknowledge support from the Universitat Politécnica de Valéncia grant PAID-06-11 Ref: 2087.

\section{References}

[1] CDC. H1N1 flu. Center for Disease Control and Prevention Website. Available from:http://www.cdc.gov/h1n1flu/.

[2] S.M. Tracht, S.Y. Del Valle, and J.M. Hyman. Mathematical modeling of the effectiveness of facemasks in reducing the spread of novel influenza A (H1N1). PLoS One, 5(2):e9018, 2010.

[3] S. Takeuchi and Y. Kuroda. Predicting spread of new pandemic swine-origin influenza $\mathrm{A}(\mathrm{H} 1 \mathrm{~N} 1)$ in local mid-size city: evaluation of hospital bed shortage and effectiveness of vaccination. Nippon Eiseigaku Zasshi, 65(1):48-52, 2010.

[4] G. F. Webb, Y-H. Hsieh, J. Wu, and M. J. Blaser. Pre-symptomatic influenza transmission, surveillance, and school closings: Implications for novel influenza A (H1N1). Math. Model. Nat. Phenom., 5(3):191-205, 2010.

[5] Sara Y Del Valle, Susan M Mniszewski, and James M Hyman. Modeling the impact of behavior changes on the spread of pandemic influenza. In Modeling the Interplay Between Human Behavior and the Spread of Infectious Diseases, pages 59-77. 2013.

[6] Gerardo Chowell, Hiroshi Nishiura, and Luis M.A Bettencourt. Comparative estimation of the reproduction number for pandemic influenza from daily case notification data. Journal of The Royal Society Interface, 4(12):155-166, 2007.

[7] Xuhui Tan, Lingling Yuan, Jingjing Zhou, Yinan Zheng, and Fen Yang. Modeling the initial transmission dynamics of influenza A1̂N1 in Guangdong Province, China. International Journal of Infectious Diseases, 17(7):e479 e484, 2013.

[8] S. Towers and Z. Feng. Pandemic H1N1 influenza: predicting the course of a pandemic and assessing the efficacy of the planned vaccination programme in the United States. Euro Surveill., 14(41):1-3, 2009.

[9] J.B.S. Ong, M.I.C. Chen, A.R. Cook, H.C. Lee, V.J. Lee, R.T.P. Lin, P.A. Tambyah, and L.G. Goh. Real-time epidemic monitoring and forecasting of H1N1-2009 Using Influenza-Like Illness from General Practice and Family Doctor Clinics in Singapore. PLoS ONE, 5(4):e10036, 2010.

[10] S. Towers and G. Chowell. Impact of weekday social contact patterns on the modeling of influenza transmission, and determination of the influenza latent period. Journal of Theoretical Biology, 312(0):87 - 95, 2012.

[11] A. Cori, A.J. Valleron, F. Carrat, G. Scalia Tomba, G. Thomas, and P.Y. Bolle. Estimating influenza latency and infectious period durations using viral excretion data. Epidemics, 4(3):132 - 138, 2012. 
[12] G. Katriel, R. Yaari, A. Huppert, U. Roll, and L. Stone. Modelling the initial phase of an epidemic using incidence and infection network data: 2009 H1N1 pandemic in Israel as a case study. Journal of The Royal Society Interface, 8(59):856-867, 2011.

[13] L.A.N. Amaral and J. Ottino. Complex networks - augmenting the framework for the study of complex systems. European Physical Journal B, 38(2):147-162, 2004 .

[14] G. Witten and G. Poulter. Simulations of infectious diseases on networks. Computers in Biology and Medicine, 37(2):195-205, 2007.

[15] L. Acedo, J.-A. Moran̂o, R.-J. Villanueva, J. Villanueva-Oller, and J. DíezDomingo. Using random networks to study the dynamics of respiratory syncytial virus (RSV) in the Spanish region of Valencia. Mathematical and Computer Modelling, 54(78):1650 - 1654, 2011.

[16] Håkan Andersson and Tom Britton. Stochastic epidemic models and their statistical analysis, volume 151. Springer New York, 2000.

[17] Silvia Rausanu and Crina Grosan. A hierarchical network model for epidemic spreading. Analysis of A/H1N1 virus spreading in Romania. Applied Mathematics and Computation, 233(0):39 - 54, 2014.

[18] L. Acedo, J.-A. Moran̂o, and J. Díez-Domingo. Cost analysis of a vaccination strategy for respiratory syncytial virus (RSV) in a network model. Mathematical and Computer Modelling, 52(78):1016 - 1022, 2010.

[19] Eduardo Massad, Stefen Ma, Mark Chen, Cláudio Jos Struchiner, Nico Stollenwerk, and Maíra Aguiar. Scale-free network of a dengue epidemic. Applied Mathematics and Computation, 195(2):376 - 381, 2008.

[20] G González-Parra, L. Acedo, R.-J. Villanueva Micó, and A.-J. Arenas. Modeling the social obesity epidemic with stochastic networks. Physica A: Statistical Mechanics and its Applications, 389(17):3692-3701, 2010.

[21] Simon M. Firestone, Robert M. Christley, Michael P. Ward, and Navneet K. Dhand. Adding the spatial dimension to the social network analysis of an epidemic: Investigation of the 2007 outbreak of equine influenza in Australia. Preventive Veterinary Medicine, 0(0):In press, 2012.

[22] Chris Groendyke, David Welch, and David R. Hunter. Bayesian inference for contact networks given epidemic data. Scandinavian Journal of Statistics, 38(3):600-616, 2011.

[23] Sandro Azaele, Amos Maritan, Enrico Bertuzzo, Ignacio Rodriguez-Iturbe, and Andrea Rinaldo. Stochastic dynamics of cholera epidemics. Phys. Rev. E, 81:051901, 2010.

[24] Carlos J. Dommar, Rachel Lowe, Marguerite Robinson, and Xavier Rod. An agent-based model driven by tropical rainfall to understand the spatio-temporal heterogeneity of a chikungunya outbreak. Acta Tropica, 129(0):61 - 73, 2014. 
[25] B. Oksendal. Stochastic Differential Equations. Springer, New York, 1995.

[26] Ekaitz Zulueta Guerrero, Asier González González, Jose Manuel Lopez-Guede, and Isidro Calvo Gordillo. Simulación basada en SMA de sistemas originalmente representados con EDO. Revista Iberoamericana de Automática e Informática Industrial RIAI, 8(4):323 - 333, 2011.

[27] Nicholas A. Christakis and James H. Fowler. The Spread of Obesity in a Large Social Network over 32 Years. N. Engl. J. Med., 357(4):370-379, 2007.

[28] S. Eubank, V. S. Anil Kumar, M. V. Marathe, A. Srinivasan, and N. Wang. Structure of social contact networks and their impact on epidemics. DIMACS Series in Discrete Mathematics and Theoretical Computer Science, 70:181-214, 2006.

[29] Gilberto C. González-Parra, R.J. Villanueva, and Lupe Segovia. Diámica del virus pandémico AH1N1/09 en la población de Venezuela. Revista Interciencia, 37(4), 2012.

[30] Gilberto González-Parra, A. Arenas, and Lupe Segovia Diego F. Aranda. Modeling the epidemic waves of AH1N1/09 influenza around the world. Spatial and Spatio-temporal Epidemiology, 2:219-226, 2011.

[31] J. Kennedy and R. Eberhart. Particle swarm optimization. In Neural Networks, 1995. Proceedings., IEEE International Conference on, volume 4, pages 1942 -1948 vol.4, 1995.

[32] D. Bratton and J. Kennedy. Defining a standard for particle swarm optimization. In Swarm Intelligence Symposium, 200\%. SIS 200\%. IEEE, pages 120-127, 2007.

[33] Heath A Kelly, Geoff N Mercer, James E Fielding, Gary K Dowse, Kathryn Glass, Dale Carcione, Kristina A Grant, Paul V Effler, and Rosemary A Lester. Pandemic (h1n1) 2009 influenza community transmission was established in one australian state when the virus was first identified in north america. PloS one, 5(6):e11341, 2010.

[34] K. Glass, G. N. Mercer, H. Nishiura, E. S. McBryde, and N. G. Becker. Estimating reproduction numbers for adults and children from case data. Journal of The Royal Society Interface, 8(62):1248-1259, 2011.

[35] Vivek Charu, Gerardo Chowell, Lina Sofia Palacio Mejia, Santiago EchevarraZuno, Vctor H. Borja-Aburto, Lone Simonsen, Mark A. Miller, and Ccile Viboud. Mortality burden of the A/H1N1 pandemic in Mexico: A comparison of deaths and years of life lost to seasonal influenza. Clinical Infectious Diseases, 53(10):985-993, 2011.

[36] Min Ouyang, Ming-hui Yu, Gao-xia Wang, and En-jie Luan. Effects of new individuals addition and initial condition on epidemic spreading. Journal of System Simulation, 10:053, 2008. 
[37] F.G. Hayden, R. Fritz, M.C. Lobo, W. Alvord, W. Strober, and S.E. Straus. Local and systemic cytokine responses during experimental human influenza A virus infection. relation to symptom formation and host defense. J Clin Invest, 101(3):643649, 1998.

[38] S. Leekha, N.L. Zitterkopf, M.J. Espy, T.F. Smith, R.L. Thompson, and P. Sampathkumar. Duration of influenza A virus shedding in hospitalized patients and implications for infection control. Infect Control Hosp Epidemiol, 28(9):10711076, 2007.

[39] Fabrice Carrat, Elisabeta Vergu, Neil M. Ferguson, Magali Lemaitre, Simon Cauchemez, Steve Leach, and Alain-Jacques Valleron. Time lines of infection and disease in human influenza: a review of volunteer challenge studies. Am J Epidemiol, 167:775-785, 2008.

[40] J.A. Nelder and R. Mead. A simplex method for function minimization. The Computer Journal, 7:308-313, 1964.

[41] W.H. Press, B.P. Flannery, S.A. Teukolsky, and W. Vetterling. Numerical Recipes: The Art of Scientic Computing. Cambridge Univ. Press, 1986.

[42] Gilberto González-Parra, Abraham J. Arenas, and Benito M. ChenCharpentier. A fractional order epidemic model for the simulation of outbreaks of influenza A(H1N1). Mathematical Methods in the Applied Sciences, page In press, 2013.

[43] Yang Wang, Deepayan Chakrabarti, Chenxi Wang, and Christos Faloutsos. Epidemic spreading in real networks: An eigenvalue viewpoint. Reliable Distributed Systems, IEEE Symposium on, 0:25, 2003.

[44] J.L. Herrera and G.C. González-Parra. Modelado de enfermedades contagiosas mediante una clase de redes sociales dinámicas. Revista de la Facultad de Ingeniería Universidad Central de Venezuela, 27(2):15-20, 2013. 